\title{
Characterization of Structure and Property of the Monocarboxyl Bamboo Pulp Fibers
}

\author{
Zhaofang Du, Liling Zhang, Yunhui Xu \\ College of Textile Engineering and Art, Anhui Agricultural University, Hefei, China \\ Email: zhanglilingblue@163.com
}

Received 5 March 2015; accepted 28 March 2015; published 31 March 2015

Copyright (C) 2015 by authors and Scientific Research Publishing Inc.

This work is licensed under the Creative Commons Attribution International License (CC BY). http://creativecommons.org/licenses/by/4.0/

(c) (i) Open Access

\begin{abstract}
The oxidized bamboo pulp fiber yarns were prepared by the $\mathrm{HNO}_{3} / \mathrm{H}_{3} \mathrm{PO}_{4}-\mathrm{NaNO}_{2}$ oxidation system. The effects of the oxidation concentration and reaction time on the weight loss, the carboxyl content and the breaking strength of the bamboo pulp fiber yarns were studied and the aggregation structure was investigated by Fourier transform infrared, X-ray and scanning electron microscopy. The results revealed an increase in carboxyl content and a decrease in breaking strength of oxidized bamboo pulp fiber yarns with increasing concentration of oxidant and reaction time. The breaking strength of oxidized bamboo fiber yarns was damaged seriously once the reaction time was more than 120 min or the concentration of oxidant was greater than $1.0 \%$. The crystallinity of bamboo pulp fibers increased slightly under low oxidation degree but decreased with higher oxidation degree.
\end{abstract}

\section{Keywords}

Bamboo Pulp Fiber, Selective Oxidation, $\mathrm{HNO}_{3} / \mathrm{H}_{3} \mathrm{PO}_{4}-\mathrm{NaNO}_{2}$ System, Structure, Property

\section{Introduction}

Bamboo pulp fiber is generated cellulose fiber and is prepared by viscose spinning process or Lyocell spinning process with bamboo as raw material, and the bamboo pulp fiber currently used mainly on the domestic market is made by viscose spinning process [1]. Bamboo pulp fiber prepared by this process has favorable dyeability; bamboo pulp fiber fabric has strong moisture absorption and desorption and good permeability; but the breaking strength would decrease after moisture absorption and the shrinkage of bamboo pulp fiber fabric is large, shape retention is poor and fabric wrinkles easily [2] [3]. And partial oxidation of bamboo pulp fiber is an effective way to improve the property of fiber.

Selective oxidation of cellulose is the reaction leads the C2, C3 or C6 hydroxyl in the cellulose macromolecular to aldehyde group, ketone group or carboxyl group. It makes oxidation cellulose own the maternal 
attributes and can combine with the other substances and increase its uses [4] [5]. Wang Juhua et al. used sodium periodate to oxidize C2, C3 hydroxyl in bamboo pulp fiber and characterized the performance of oxidized bamboo pulp fibe [6]. But reports about the use of selective oxidation of bamboo pulp fiber by $\mathrm{HNO}_{3} / \mathrm{H}_{3} \mathrm{PO}_{4^{-}}$ $\mathrm{NaNO}_{2}$ system [7] are rarely.

In this study, we oxidized the bamboo pulp fiber yarns selectively by $\mathrm{HNO}_{3} / \mathrm{H}_{3} \mathrm{PO}_{4}-\mathrm{NaNO}_{2}$ system and studied the effects of oxidation concentration and reaction time on the structure and property of fiber, so as to provide the basis for the functional modification on bamboo pulp fiber.

\section{Materials and Methods}

\subsection{Materials}

Bamboo pulp fiber yarns (28 tex); $\mathrm{HNO}_{3}(68 \% \mathrm{w} / \mathrm{v}), \mathrm{H}_{3} \mathrm{PO}_{4}(85 \% \mathrm{w} / \mathrm{v}), \mathrm{NaNO}_{2}$, ethanol, $\mathrm{HCl}$, calcium acetate and $\mathrm{NaOH}$ used for the following investigations were analytical grade.

\subsection{Methods}

\subsubsection{Preparation of Oxidized Bamboo Pulp Fiber Yarns}

Nitric acid and phosphoric acid were mixed in 2:1 (v/v) ratio. According to the liquor ratio 1:30, a certain quality bamboo pulp fiber yarn was added to the solution of acid mixture. Once the yarns was completely soaked, a certain mass sodium nitrite were added all at once, the concentration of sodium nitrite were $0.6 \%, 1.0 \%, 1.4 \%$ and $1.8 \%(\mathrm{~W} / \mathrm{V})$ respectively. The reaction mixture was allowed to react at room temperature, under dark condition and with occasional stirring for 30,60,120, 180 or 300 min. Once the oxidation reaction completed, the bamboo pulp fiber yarns were washed with deionized water until the lotion showed a $\mathrm{pH}$ of about 4 . Then the yarn was soaked in a certain concentration of ethanol for 20 min. Finally it was washed with deionized water and then dried at $60^{\circ} \mathrm{C}$.

\subsubsection{Determination of Carboxyl Content}

It was performanced according to the method described in the United States Pharmacopoeia [8]. Approximately $0.5 \mathrm{~g}$ of the sample, cut into small pieces, was accurately weighed and vibrated in $50 \mathrm{ml}$ of a $2 \%(\mathrm{w} / \mathrm{w})$ calcium acetate solution for $30 \mathrm{~min}$ with ultrasonic oscillator. The mixture was titrated with $0.1 \mathrm{~mol} / \mathrm{L} \mathrm{NaOH}$ using phenolphthalein as an indicator.

$$
\text { Carboxyl groups }(\%)=\frac{\mathrm{N} \times \mathrm{V} \times \mathrm{MW}_{\mathrm{COOH}}}{\mathrm{m} \times(1-\mathrm{w} / 100)} \times 100 \%
$$

where $\mathrm{N}$ is the normality of $\mathrm{NaOH}, \mathrm{V}$ is the volume of $\mathrm{NaOH}$ solution in ml consumed in titration and after correcting for the blank, $\mathrm{m}$ is the quality of yarns, and $\mathrm{w}$ is the moisture regain of bamboo pulp fiber.

\subsubsection{Determination of Weight Loss}

The weight of original bamboo pulp fiber yarns was $\mathrm{m} 1$, the weight of oxidized bamboo pulp fiber yarns is $\mathrm{m} 2$.

$$
\text { Weight loss }(\%)=\frac{m 1-m 2}{m 1} \times 100
$$

\subsubsection{Fourier-Transform Infrared Spectroscopy}

The FT-IR spectra were recorded on a Nicolette is 50 FT-IR spectrophotometer using the traditional transmission technique for $\mathrm{KBr}$ pellets. $\mathrm{KBr}$ pellets were prepared by mixing $1-2 \mathrm{mg}$ of the bamboo pulp fiber powders with $200 \mathrm{mg}$ of $\mathrm{KBr}$ in a ball mixer and then pelleted.

\subsubsection{Scanning Electron Microscopy (SEM)}

The surface of varying degrees of oxidized bamboo pulp fibers were observed on a Sirion 200 scanning electron microscopy.

\subsubsection{XRD Patterns}

XRD patterns of bamboo pulp fiber powders were determined by a model MXPAHF X-ray diffraction system at 
voltage of $40 \mathrm{kv}$, current of $30 \mathrm{~mA}$, scan rate of $4^{\circ} / \mathrm{min}$ and measurements over $5^{\circ}-50^{\circ} 2 \theta$.

\subsubsection{Determination of Breaking Strength}

The breaking strength of bamboo pulp fiber yarns were determined at an effective gauge length of $200 \mathrm{~mm}$ and extension rate of $500 \mathrm{~mm} / \mathrm{min}$ performed on a YG021-A electron single yarn strength tester.

\section{Results and Discussion}

\subsection{Carboxyl Content and Weight Loss of Oxidized Bamboo Pulp Fiber Yarns}

The oxidation degree of bamboo pulp fiber yarns were characterized by carboxyl content and results shows in Table 1. The carboxyl contents of bamboo pulp fiber yarns presented in Table 1 were $0.37 \%$ and $1.09 \%$ respectively after being oxidized $60 \mathrm{~min}$ and $120 \mathrm{~min}$ in the solution of acid mixture with the concentration of $0.6 \%$ $\mathrm{NaNO}_{2}$, were close to the carboxyl contents of bamboo pulp fiber yarns, oxidized for $30 \mathrm{~min}$ in the solution of acid mixture with the concentration of $1.0 \%$ and $1.4 \% \mathrm{NaNO}_{2}$. It indicates that with the extension of reaction time, the carboxyl content of oxidized bamboo pulp yarns increased. To obtain the same carboxyl content of oxidized bamboo pulp fiber yarns, the higher oxidation concentration needed the less reaction time.

Figure 1 shows the weight loss of bamboo pulp fiber yarns in different oxidation condition. As Figure 1 shows, with the increasing of oxidation concentration and extension of reaction time, the weight loss of bamboo pulp fiber yarns increased. When the reaction time is more than $120 \mathrm{~min}$, the weight loss of bamboo pulp fiber yarns increased obviously.

It is plausible that during reaction the C6 hydroxyl of bamboo pulp fiber was oxidized to be carboxyl by $\mathrm{HNO}_{3} / \mathrm{H}_{3} \mathrm{PO}_{4}-\mathrm{NaNO}_{2}$ system but the end units of the cellulose chains were hydrolyzed. Therefore, the weight of bamboo pulp fiber yarns decreased. The oxidation reaction, using $\mathrm{HNO}_{3} / \mathrm{H}_{3} \mathrm{PO}_{4}-\mathrm{NaNO}_{2}$ system as the solution, occurred gradually from amorphous region to crystalline region [9]. In the early stage of oxidation reaction, the end units of the cellulose chains in the amorphous regions are more susceptible to hydrolysis, which caused the weight loss of bamboo pulp fibers. With the extension of reaction time, the oxidation gases spread to the amorphous regions, the hydrogen bond and van der waals between the cellulose chains were decreased or destroyed, the structure of crystalline regions were damaged and the hydrolysis of the end units of the cellulose chains increased, which lead to the dissolution loss of weight. Therefore, the weight of bamboo pulp fiber yarns decreased more seriously. Also with the increase of oxidation concentration, the effects of diffusion and penetration of oxidation gases $\mathrm{NO}_{2}$ and $\mathrm{NO}$ to the interior of bamboo pulp fibers and the accessibility of oxidant to the C6 hydroxyl increased. Therefore, with the increase of oxidation concentration, the carboxyl content of bamboo pulp fiber yarns increased but the weight decreased.

\subsection{FT-IR Spectra Analysis}

Figure 2 shows the FT-IR spectra of the pure bamboo pulp fibers and the oxidized fibers with different carboxyl contents. Cure b, c, d and e are the spectrum cures of oxidized bamboo pulp fibers. Compared with cure a, the spectrum cure of the pure bamboo pulp fibers, a new characteristic absorption band appeared at around 1731.7 $\mathrm{cm}^{-1}, 1733.6 \mathrm{~cm}^{-1}, 1735.6 \mathrm{~cm}^{-1}$ and $1741.4 \mathrm{~cm}^{-1}$ respectively due to the stretching vibration of the $\mathrm{C}=\mathrm{O}$ double bond [10]. And with the increase of oxidation degree, the carboxyl characteristic peaks become higher. This indicates that the $\mathrm{C} 6$ hydroxyl were oxidized to be carboxyl partly by $\mathrm{HNO}_{3} / \mathrm{H}_{3} \mathrm{PO}_{4}-\mathrm{NaNO}_{2}$ system.

Table 1. Carboxyl contents in different oxidation conditions (\%).

\begin{tabular}{|c|c|c|c|c|}
\hline \multirow{2}{*}{ Time/min } & \multicolumn{4}{|c|}{ Concentration of $\mathrm{NaNO}_{2}(\%)$} \\
\hline & 0.6 & 1.0 & 1.4 & 1.8 \\
\hline 30 & 0.27 & 0.32 & 0.51 & 0.85 \\
\hline 60 & 0.37 & 0.89 & 1.06 & 1.66 \\
\hline 120 & 1.09 & 1.41 & 2.35 & 3.58 \\
\hline 180 & 1.33 & 2.22 & 3.43 & 5.30 \\
\hline 300 & 2.75 & 4.40 & 6.80 & 8.19 \\
\hline
\end{tabular}




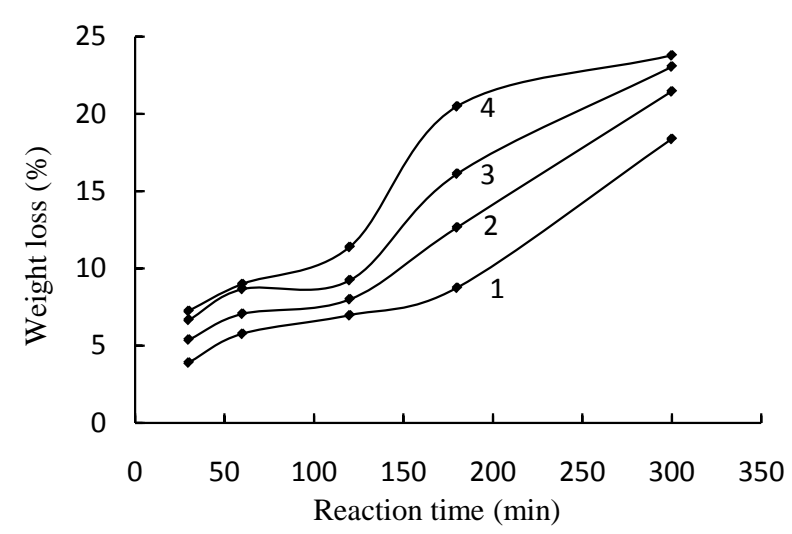

Figure 1. Weight loss of oxidized bamboo pulp fiber yarns: (1) $\mathrm{NaNO}_{2}, 0.6 \%$, (2) $\mathrm{NaNO}_{2}, 1.0 \%$, (3) $\mathrm{NaNO}_{2}, 1.4 \%$, (4) $\mathrm{NaNO}_{2}, 1.8 \%$.

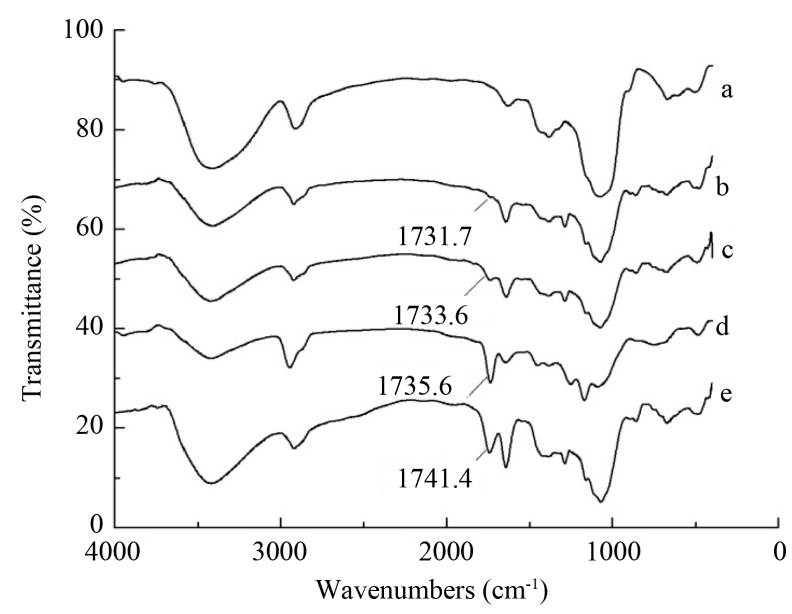

Figure 2. FT-IR spectra of (a) pure bamboo pulp fiber, (b) bamboo pulp fiber with $0.32 \%$ carboxyl content, (c) bamboo pulp fiber with $2.22 \%$ carboxyl content, (d) bamboo pulp fiber with $3.58 \%$ carboxyl content, (e) bamboo pulp fiber with $8.19 \%$ carboxyl content.

\subsection{Scanning Electron Microscopic Analysis}

The surface of the bamboo pulp fibers before and after oxidizing treatment were observed by SEM. The surface of the pure bamboo pulp fiber shown in Figure 3(a) is smooth and there are many shade stripes. In Figures 3(b)-(e), attachments are found on the surface and with the increase of carboxyl content, twists are found, stripes become less apparent and even disappeared. It illustrates that the surface of bamboo pulp fibers were eroded by the $\mathrm{HNO}_{3} / \mathrm{H}_{3} \mathrm{PO}_{4}-\mathrm{NaNO}_{2}$ system and bamboo pulp fibers contracted.

\subsection{XRD Diffraction Analysis}

Figure 4 shows the XRD cures of the bamboo pulp fibers before and after oxidation. The peaks of cure a, b, c and $\mathrm{d}$ are located near $2 \theta=12.5^{\circ}, 20.2^{\circ}$ and $22.2^{\circ}$, which were the characteristic diffraction peaks of the cellulose II [11]. This indicates that the crystalline structure of bamboo pulp fiber was not altered.

Meanwhile, the crystalline degree of bamboo pulp fiber with $0.32 \%$ carboxyl content increased slightly. This is because under low oxidation degree, the end units of the cellulose chains in amorphous zone were mainly eroded away and the rate of amorphous zone decreased. However, with higher oxidation degree, the crystalline degree of bamboo pulp fibers decreased. This can be explained in this way, with the increase of oxidation degree, 

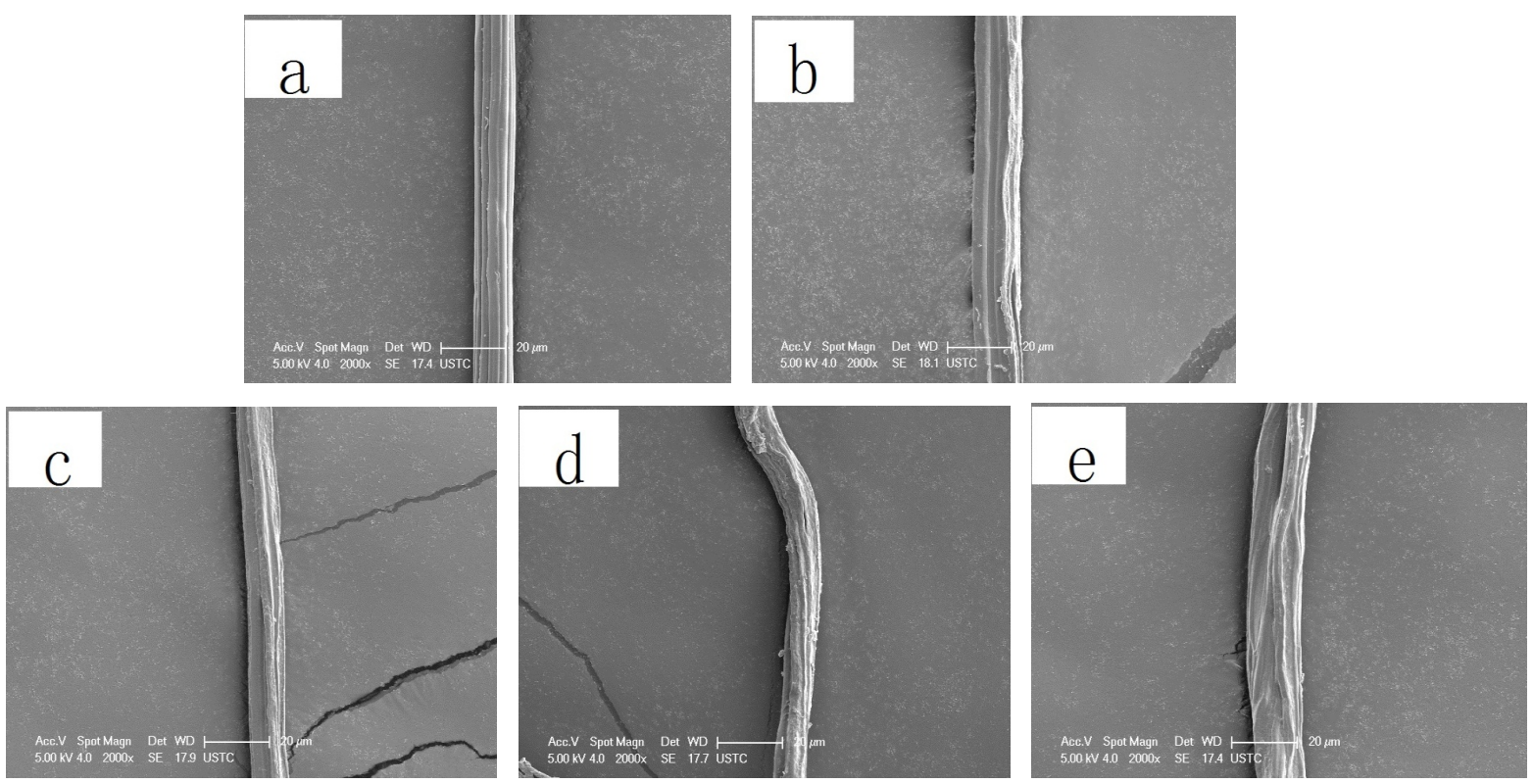

Figure 3. SEM photographs of (a) pure bamboo pulp fiber, (b) bamboo pulp fiber with $0.32 \%$ carboxyl content, (c) bamboo pulp fiber with $2.22 \%$ carboxyl content, (d) bamboo pulp fiber with $3.58 \%$ carboxyl content, (e) bamboo pulp fiber with $8.19 \%$ carboxyl content.

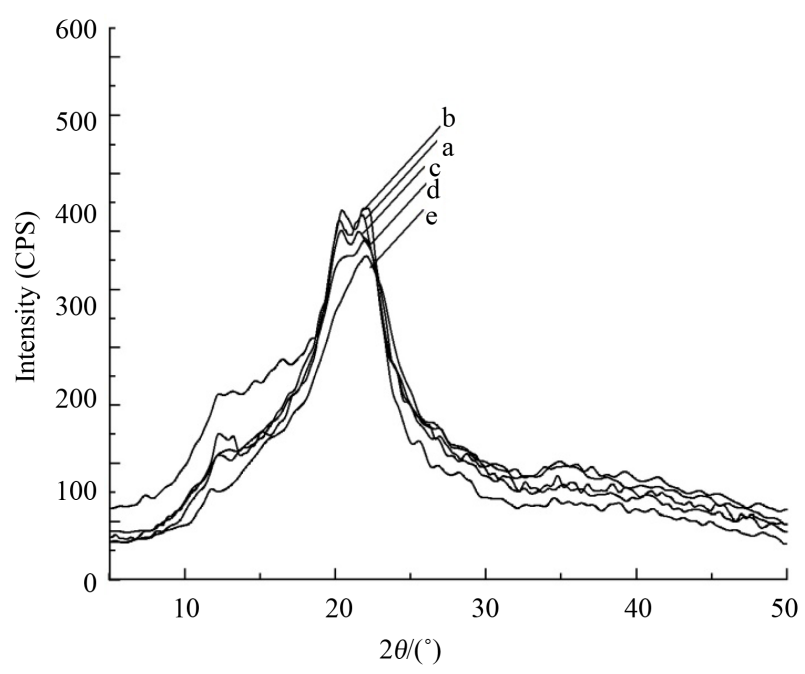

Figure 4. Powder X-ray diffraction patterns of (a) pure bamboo pulp fiber, (b) bamboo pulp fiber with 0.32\% carboxyl content, (c) bamboo pulp fiber with $2.22 \%$ carboxyl content, (d) bamboo pulp fiber with $3.58 \%$ carboxyl content, (e) bamboo pulp fiber with $8.19 \%$ carboxyl content.

oxidant spread to the amorphous zone and the force between cellulose chains were destroyed, the hydrolysis of the end units of the cellulose chains increased, the structure of crystalline regions was damaged and the crystalline degree of bamboo pulp fibers decreased. When the carboxyl content increased to $8.19 \%$, diffraction peak near $20.2^{\circ}$ disappeared and diffraction peak near $22.2^{\circ}$ become weaker and broader. It is possible that with the increase of oxidation degree, the hydrogen bonds between cellulose chains were destroyed, most of crystalline regions of bamboo pulp fibers transformed into amorphous region and the structure of crystallinity was damaged seriously.

\subsection{The Breaking Strength Test}

Figure 5 shows the breaking strength of bamboo pulp fiber yarns oxidized by different $\mathrm{NaNO}_{2}$ concentration of 


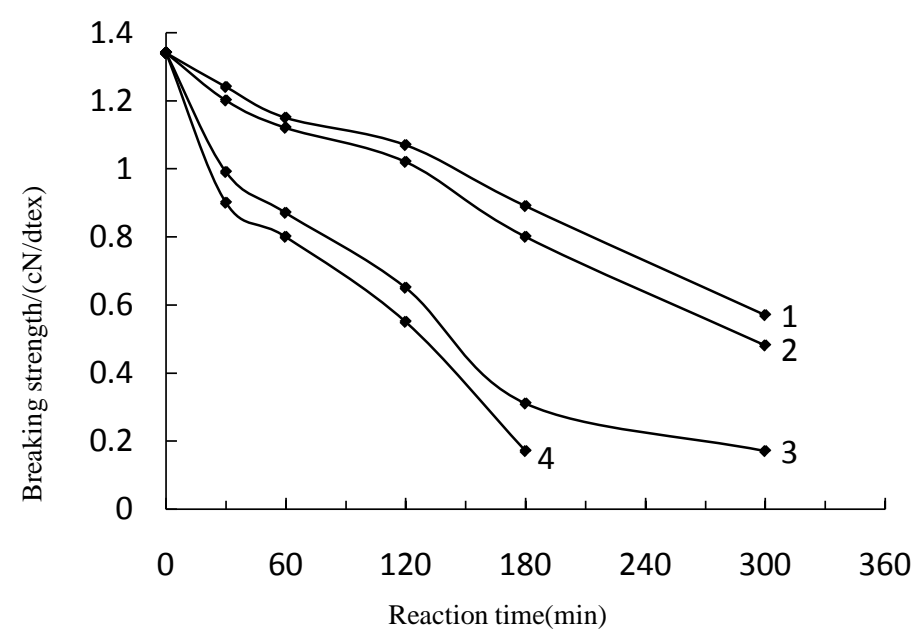

Figure 5. Breaking strength of oxidized bamboo pulp fiber yarns: (1) $\mathrm{NaNO}_{2}, 0.6 \%$, (2) $\mathrm{NaNO}_{2}, 1.0 \%$, (3) $\mathrm{NaNO}_{2}, 1.4 \%$, (4) $\mathrm{NaNO}_{2}, 1.8 \%$.

acid mixture. With the increase of oxidation concentration or reaction time, the breaking strength of bamboo pulp fiber yarns decreased. The breaking strength of bamboo pulp fiber yarns oxidized by the solution, whose concentration of $\mathrm{NaNO}_{2}$ was lower than $1.0 \%$, for less than 120 min, could keep more than $75 \%$ compared with the breaking strength of pure bamboo pulp fiber yarns. However, the breaking strength was damaged obviously once the oxidation concentration was higher than $1.0 \%$ or the reaction time longer than 120 min. The yarns become hard and brittle and the breaking strength could not be tested even when the oxidation concentration increased to $1.8 \%$ and reaction time exceeded $180 \mathrm{~min}$.

The breaking strength of yarns were damaged slightly is plausible that in the early stage of oxidation reaction, oxidant could permeate the amorphous regions quickly but had no apparent influence on the crystalline regions and low concentration of oxidant would bring few hydrolysis of the end units of the cellulose chains. As the oxidation reaction progressed, the oxidant spread to the crystalline regions, the disruption of hydrogen bonds between cellulose chains and the hydrolysis of the end units of the cellulose chains progressively increased, causes a linear decrease in the degree of crytallinity and a serious damage in the breaking strength.

\section{Conclusions}

1) Increasing the oxidation concentration of acid mixture or reaction time could raise the carboxyl content of oxidized bamboo pulp fiber yarns but cause the increase of weight loss. The weight loss of bamboo pulp fiber yarns increased obviously once the reaction time exceeded 120 min.

2) The breaking strength of oxidized bamboo pulp fiber yarns under the condition that oxidation concentration was lower than $1.0 \%$ and reaction time less than 120 min could maintain more than $75 \%$ compared with the breaking strength of pure bamboo pulp fiber yarns. However, the breaking strength was damaged obviously once the oxidation concentration was higher than $1.0 \%$ or the reaction time was longer than $120 \mathrm{~min}$. The breaking strength could not be tested when the oxidation concentration increased to $1.8 \%$ and reaction time exceeded $180 \mathrm{~min}$.

3) FT-IR spectra indicated that carboxyl generated in the macromolecule of bamboo pulp fibers during oxidation process. The crystalline degree increased slightly under low oxidation degree but with the increase of oxidation degree, the crystalline degree decreased gradually.

\section{References}

[1] Liu, H. and Lv, B. (2013) Performance, Process and Development Prospects of Bamboo Fiber. Hebei Chemical Industry, 2, 57-59.

[2] Li, R.Z., Liu, Y.L. and Sun, Z.B. (2004) Analysis on Performance of Bamboo Fiber. Journal of Textile Research, 3, 76-77.

[3] Ye, Y.J. and Yuan, X.H. (2011) Analysis of Mechanical Properties of Bamboo Pulp Fibers. China Fiber Inspection, 17, 
82-84.

[4] Li, L., Zhao, S. and Hu, H.Q. (2009) Development in Oxidative System of Cellulose. Journal of Cellulose Science Technology, 3, 59-64.

[5] Geng, C.Z., Xia, Y.Z. and Jin, F.Y. (2012) Research on the Selective Oxidation and Development Trend of Cellulose. Journal of Functional Materials, 15, 1976-1980.

[6] Kumar, V. and Yang, T.R. (2002) $\mathrm{HNO}_{3} / \mathrm{H}_{3} \mathrm{PO}_{4}-\mathrm{NaNO}_{2}$ Mediated Oxidation of Cellulose Preparation and Chararcterization of Bioabsorbable Oxidized Celluloses in High Yields and with Different Levels of Oxidation. Carbohydrate Polymers, 4, 403-412. http://dx.doi.org/10.1016/S0144-8617(01)00290-9

[7] The United States Pharmacopeia Convention, Inc. (2007) United States Pharmacopeia. XX Ш Revision. Port City Press, Baltimore, 1687.

[8] Xu, Y.H., Lin, H. and Chen, Y.Y. (2006) Aggregated Structure of Cotton Fiber Oxidized Selectively with Sodium Periodate. Journal of Textile Research, 11, 1-5.

[9] Wang, J.H., Chen, Y.Y., Lin, H., et al. (2007) Structure and Properties of the Oxidized Bamboo Pulp Fiber. Journal of Nantong University (Natural Science), 4, 54-57.

[10] Wang, L., Xiang, B.R. and Zou, Q.G. (2009) Preparation of the Oxidized Cellulose and the Related Study on Its Structure and Performance. Progress in Pharmaceutical Sciences, 8, 365-369.

[11] Ma, X.J., Huang, L.L., Chen, L.H., et al. (2012) Determination Methods for Crystallinity Cellulose. Paper Science \& Technology, 2, 75-78. 